\title{
BMJ Open Are the knowledge of non-malignant asbestos-related diseases and lung function impairment differentially associated with psychological well- being? A cross-sectional study in formerly asbestos-exposed workers in Germany
}

Jessica Lang (1) , Michael K Felten, Thomas Kraus

To cite: Lang J, Felten MK, Kraus T. Are the knowledge of non-malignant asbestosrelated diseases and lung function impairment differentially associated with psychological well-being? A cross-sectional study in formerly asbestos-exposed workers in Germany. BMJ Open 2019;9:e030094. doi:10.1136/ bmjopen-2019-030094

- Prepublication history for this paper is available online. To view these files, please visit the journal online (http://dx.doi. org/10.1136/bmjopen-2019030094).

Received 26 February 2019 Revised 18 September 2019 Accepted 03 October 2019

Check for updates

(C) Author(s) (or their employer(s)) 2019. Re-use permitted under CC BY-NC. No commercial re-use. See rights and permissions. Published by BMJ.

Institute of Occupational, Social and Environmental Medicine, RWTH Aachen University, Aachen, Germany

Correspondence to

Dr Jessica Lang;

jlang@ukaachen.de

\section{ABSTRACT}

Objectives The knowledge of past asbestos exposure may lead to chronic psychological strain. In addition, the information about an increased cancer risk can place a psychological burden on individuals triggering mental health symptoms of depression or anxiety. This applies in particular to individuals with non-malignant asbestosrelated disease (ARD) such as lung fibrosis and pleural thickening with or without lung function impairment. ARDs with or without lung function impairment may develop even years after exposure cessation. Therefore, the aim of the present study was to test for our cohort whether non-malignant ARD and lung function impairment have differential effects on mental health and psychological strain.

Design Cross-sectional study.

Participants and setting Overall, 612 male participants (mean age $=66.2$ years, $\mathrm{SD}=9.5$ ) attending a surveillance programme for ARDs received routine examinations including lung function testing (24\% refused to fill in the psychological questionnaire) at a German university hospital study centre from August 2008 to August 2013. Outcome measures Using multiple hierarchical regression analysis, ARD diagnosis and lung function impairment were used to predict psychological health as measured with validated questionnaires for depression and anxiety. Psychological strain was operationalised by intrusive thoughts and specific fear of cancer.

Results The strongest predictor for mental health was obstructive functional impairment (eg, anxiety: $\beta=0.22$, $p<0.001)$. Psychological strain was predicted by the presence of a non-malignant ARD (eg, intrusive thoughts: $\beta=0.17, p=0.003$ ).

Conclusions The presence of mental health symptoms is associated with ventilation disturbances, whereas the knowledge of an already initiated morphological change — caused by asbestos exposure-is primarily associated with psychological strain. Specifically, the affected individuals are more prone to intrusive thoughts and specific fear of asbestos-related cancer. As an
Strengths and limitations of this study

- The main strength of the study is that it takes a differentiated look at psychological well-being after hazardous substance exposure by considering data from psychological self-report surveys and objective medical examinations.

- Combining medical data with psychological surveys reduces the potential for monomethod bias in study results.

- Data generalisability was limited due to all male gender and incomplete data in the psychological survey, because of increased age and health impairments of the present study population.

- Future research should prospectively follow-up on a younger age cohort-including male and female participants - that has been exposed to other hazardous substances to understand the relative strength of the influencing factors (knowledge regarding health risk vs experienced functional impairments) on psychological well-being.

implication, physicians should be sensitised about possible consequences of risk communication and functional impairment to counteract excessive fear or anxiety.

\section{INTRODUCTION}

Despite the large number of formerly asbestos-exposed people worldwide, the number of research projects on the psychological consequences of past asbestos exposure is limited. ${ }^{1}$ In fact, a recent review only detected nine studies focusing on mental health of individuals (not patients) with former asbestos exposure. ${ }^{2}$ Recent studies have primarily dealt with the influence of preventive interventions like CT scan screening on mental stress. ${ }^{34}$ 
The present study intends to list possible consequences regarding psychological well-being from past asbestos exposure, differentiated according to: (1) the diagnosis of non-malignant asbestos-related diseases (ARDs) due to the past hazardous exposure and (2) the resulting health impairments potentially following an ARD.

Researchers, who studied more than the physical health of formerly asbestos-exposed workers, found an increased risk perception of asbestos workers for developing an ARD-like cancer. ${ }^{35}$ The increased health risk represents a potentially threatening information and may cause a permanent state of tension for those affected. ${ }^{5}$ This applies in particular to patients already suffering from asbestosis. ${ }^{6}$

It can be assumed that after receiving this incriminating information, individuals will undergo a psychological adaptation process to learn how to cope with the threat. ${ }^{7}$ If this adaptation process fails, emotional stress states such as depression and anxiety may occur (see International Statistical Classification of Diseases and Related Health Problems, 10th Revision, F43.2) ${ }^{8}$ In fact, past research has shown increased prevalence rates for symptoms of depression $(9.9 \%)$ and anxiety $(19.7 \%)$ in retired asbestos workers assessed with the Hospital Anxiety and Depression Scale (HADS). ${ }^{1}$ In comparison, the point prevalence for the general German male population is $4.2 \%$ for depression and $5.3 \%$ for anxiety disorders. ${ }^{9}$ Other researchers failed to find elevated levels of psychological symptoms in former asbestos workers $\left(\mathrm{eg},{ }^{5}\right)$, also not for patients with detectable radiological abnormalities. ${ }^{10}$

Besides the potential psychological consequences of the perceived health threat, especially for those workers who have already developed non-malignant asbestos-related changes of the lung or pleura, these changes may also come along with functional impairments of the lung like restriction and obstruction. ${ }^{11}$ Therefore, it needs to be disentangled, whether the reduced psychological health can only be attributed to the knowledge of a relevant exposure to a hazardous substance like asbestos or the real experienced health impairments. After all, studies analysing lung diseases with similar symptoms like chronic obstructive pulmonary disease (COPD) or idiopathic pulmonary fibrosis (IPF) also report associations for reduced psychological health. For example, in their review, Maurer and colleagues report prevalence rates in COPD for elderly patients for depression ranging from $7 \%$ to $32 \%$ measured with the HADS scale. ${ }^{12}$ For anxiety disorders they report prevalence rates even ranging from $13 \%$ to $55 \%$. Similarly, studies looking at IPF report increased prevalence rates for depression $(22.3 \%)$ and anxiety $(21.5 \%) .^{13}$

The aim of the present study was to differentially asses the importance of both (1) knowledge of an increased health risk due to past asbestos exposure manifested in non-malignant asbestos-related changes and (2) functional impairment like obstruction or restriction with regard to psychological well-being. Besides only looking at the psychological manifestations in terms of potential depression and anxiety disorders, psychological strain was also assessed in terms of specific risk of future illnesses and intrusive thoughts brought on by past exposure.

\section{METHODS}

\section{Participants and procedure}

Participants were 612 formerly asbestos-exposed male power industry workers (mean age 66.17 years, $\mathrm{SD}=9.52$ ) attending a local surveillance programme between August 2008 and August 2013, which had been initiated to improve early detection of ARDs and reduce under-reporting. ${ }^{14}$ The participants at the local study centre were part of a larger cohort $(n=8.632)$ of the Comprehensive Asbestos Research Endeavor Study (CARE Study). They were all power industry workers registered at the Central Registration Agency for Employees Exposed to Asbestos Dust in Germany. The enrolment for the early detection programme started originally in the late 1990s organised by company medical officers. ${ }^{14}$ It was based on signed statements by active or former workers, confirming that they had been occupationally exposed to asbestos fibres. To assess the cumulative individual burden of asbestos exposure, information on job titles, years of exposure and specific occupational tasks were collected by means of a specially designed self-administered questionnaire. This information was analysed in a standardised way using a computer software based on ambient monitoring data of airborne asbestos fibre concentrations at defined workplaces, stratified by specified occupational tasks and time periods. The basic reference data have been published in the format of a technical report. ${ }^{15}$ The cumulative dose of one standard fibre year was defined as an exposure during 1920 work hours through daily 8-hour shifts over 240 workdays and spread over 48 weeks with a standard airborne fibre concentration of one fibre per cubic centimetre or $1 \times 10^{6}$ fibres per cubic metre. ${ }^{14}$ The median asbestos exposure in our cohort were 8 fibre years. Among the workers, $84.5 \%$ were power generation workers, $9 \%$ were power distribution workers and $1.5 \%$ worked in gas supply. The minority of the sample were smokers $(22.3 \%)$ and a majority of $41.2 \%$ reported to be ex-smokers. The majority of participants were living together with a partner $(83.3 \%)$ and had a secondary school degree $(74.6 \%)$.

When arriving at the study centre, attendees were asked to take part in a psychological screening survey before the medical examination, which included lung function testing using a whole-body plethysmograph (MasterScreen Body, CareFusion Germany). All participants had given written informed consent before the testing. The project was approved by the ethical review board of the local university hospital (EK 043/09).

\section{Patient and public involvement}

We did not involve patients or the public in study planning or design.

\section{Lung function test}

An obstructive ventilation impairment was diagnosed if FEV1/FVC (forced expiratory volume in $1 \mathrm{~s} /$ forced vital 
capacity) was smaller than lower limit of normal (LLN, fifth percentile) using the Global Lung Function Initiative (GLI) reference values. ${ }^{16}$ The extent of obstruction was classified as mild ( $\geq 85 \%$ LLN), moderate $(<85 \%$ to $\geq 55 \%$ LLN) or severe (<55\% LLN).

A restrictive impairment was diagnosed if the total lung capacity (TLC) was smaller than the fifth percentile and FVC was smaller than the LLN according to GLI reference values. ${ }^{16}$ The extent of restrictive impairment was classified as mild ( $\geq 85 \%$ LLN), moderate $(<85 \%$ LLN and $\geq 55 \%$ LLN) or severe $(<55 \%$ LLN $)$.

\section{Measures}

Mental health: Anxiety was assessed with the 7-item subscale of the short version of the Depression Anxiety Stress Scale (DASS21-G). ${ }^{17}$ The scale captures the essential components of anxiety: physiological arousal, panic and anxiety. Based on a comprehensive study, this instrument was awarded good psychometric characteristics. ${ }^{18}$ Reliability (Cronbach's $\alpha$ ) in the present study was 0.74 .

Mental health: Depression was measured with the 7-item subscale of the DASS21-G. ${ }^{17}$ The scale records symptoms of dysphoric mood. On a 4 -step response scale $(0=\operatorname{did}$ not apply at all to me, $3=$ applied very strongly to me or most of the time), participants indicate to what extent different statements apply to them during the last week (eg, I felt depressed and sad). The scale can be used continuously as a sum scale or dichotomised with a validated cut-off value of $9 .{ }^{19}$ Reliability in the present study was 0.83 .

Psychological strain: The specific fear of asbestos-related cancer was assessed by a 1-item measure called global fear of cancer, which has been adapted for the purpose of the present study. ${ }^{20}$ On a 4-point scale, participants state to what extent they were afraid in the past week of actually having asbestos-related cancer.

Psychological strain: We measured experienced suffering with the Intrusion Subscale of the Impact of Stress Scale. ${ }^{21}$ The scale measures the stress resulting from the increased risk of developing a disease due to exposure to a carcinogenic substance. On a 4-point scale, participants indicate on 7 items the extent to which and how often they show intense thoughts and feelings due to their increased risk of illness. Reliability in the present study was 0.91 .

\section{Statistical analyses}

First, descriptive statistics are reported including a sample characteristic in relation to the frequency of medically diagnosed ventilation disturbances and psychological health symptoms of depression and anxiety. Second, the mental health of the sample is described, according to the presence of an already non-malignant asbestos-related morphological change, to initially screen whether non-malignant ARDs are associated with mental health. For this purpose, the psychological measures were dichotomised according to the cut-off score reported in the respective test manuals. Finally, the main research questions were approached with multiple hierarchical regression analyses to determine the relative impact of each predictor (ie, the knowledge of already benign ARDs vs actual lung function impairments of obstruction or restriction) on either mental health symptoms (ie, depression or anxiety) or psychological strain (ie, specific fear of cancer or intrusive thoughts). In a first step, control variables of age, education and cumulative asbestos exposure (in fibres/cubic centimetre $\times$ years) were entered into the regression equation. In a second step, the three main predictor variables (ie, benign ARDs, obstruction, restriction) were entered to the model to determine the partial regression weight for each variable in consideration of the others. The respective predictor scales are used categorically in case of the presence of obstruction or restriction in the lung function test or continuously for the psychological variables to make the maximum statistical information available. All statistical analyses were performed with SPSS for Windows version $25 .{ }^{22}$

\section{RESULTS}

The willingness to participate in the psychological screening was moderate. Out of the 612 attendees of the surveillance programme for ARDs, 147 refused to participate in the survey (24\%). Their age was $67.7(\mathrm{SD}=8.60)$ and thus significantly higher than the average age of participants providing complete surveys $(\mathrm{n}=242,39.5 \%$; mean=63.9 years; $\mathrm{SD}=10.4 ; F=24.9 ; \mathrm{p}<0.001)$. The only other significant difference in those groups was that those who refused to participate in the psychological survey also had significantly more obstructive impairments $\left(\chi^{2}=4.95\right.$, $\mathrm{p}=0.03$ ). To maximise the information, for each regression model the analyses are presented with the maximum $\mathrm{N}$ of complete datasets.

\section{Descriptive statistics}

Table 1 provides an overview of the sample's lung function results. Obstructive impairment of lung function was more frequent than restrictive impairment. We found no case of severe restriction. In addition, two individuals with mild restrictive impairment also had a moderate obstructive impairment. One participant with a mild restrictive impairment had a severe obstructive impairment. Due to the low incidence of restrictive impairments, results regarding this variable need to be considered with care.

Table 1 Distribution of ventilation impairments diagnosed with body plethysmographic lung function testing at the study centre

\begin{tabular}{llllll}
\hline \multirow{2}{*}{ Impairment } & \multicolumn{2}{l}{ Restriction } & & \multicolumn{2}{c}{ Obstruction } \\
\cline { 2 - 3 } \cline { 5 - 6 } & $\mathbf{n}$ & $\%$ & & $\mathbf{n}$ & $\%$ \\
\hline No & 597 & 97.6 & & 446 & 72.9 \\
Mild & 10 & 1.6 & & 117 & 19.1 \\
Moderate & 5 & 0.8 & & 35 & 5.7 \\
Severe & 0 & 0 & & 14 & 2.3 \\
Total & 612 & 100 & & 612 & 100 \\
\hline
\end{tabular}


Table 2 Psychological symptoms of depression and anxiety of study participants

\begin{tabular}{llllll}
\hline \multirow{2}{*}{$\begin{array}{l}\text { Psychological } \\
\text { symptoms }\end{array}$} & \multicolumn{2}{l}{$\begin{array}{l}\text { Depression } \\
\text { DASS }\end{array}$} & \multicolumn{3}{l}{$\begin{array}{l}\text { Anxiety } \\
\text { DASS }\end{array}$} \\
\cline { 2 - 3 } \cline { 5 - 6 } & $\mathbf{n}$ & $\%$ & & $\mathbf{n}$ & $\%$ \\
\hline No & 348 & 97.2 & & 328 & 89.4 \\
Yes & 10 & 2.8 & & 39 & 10.6 \\
Missing & 256 & & 247 & \\
\hline
\end{tabular}

DASS, Depression Anxiety Stress Scale.

Table 2 provides an overview of participants' psychological symptoms of depression and anxiety. With $2.8 \%$, the point prevalence for depression was low. The point prevalence of a potential anxiety disorder was $10.6 \%$. Thus, the present sample of formerly asbestos-exposed workers did not overly suffer from depression but from anxiety instead.

\section{Inferential statistics}

To understand the relevance of an already diagnosed non-malignant ARD for mental health outcomes, table 3 reports the frequency of mental disorders in the study sample in relation to a non-malignant ARD. Related to mental health, the diagnosis of a non-malignant ARD was associated with an increased risk of a possible anxiety disorder but not with an increased risk for depression.

\section{Mental health}

To differentiate between the effects of the diagnosis of a non-malignant ARD and thus a knowledge regarding an increased health risk due to former asbestos exposure and the mere presence of a ventilation impairment, table 4 presents the results of the hierarchical multiple regression analyses, which jointly consider the relevant predictors.

The diagnosis of a non-malignant ARD was not associated with any mental health symptom. When considering all predictor variables simultaneously, only the partial regression coefficient for obstructive ventilation impairment was predictive for screening measures of depression $(\beta=0.18, p=0.004)$ and anxiety $(\beta=0.22, p<0.001)$.

The diagnosis of an ARD became only relevant in the milder psychological strain variables of specific fear of cancer $(\beta=0.13, p=0.046)$ and intrusive thoughts $(\beta=0.17$, $\mathrm{p}=0.003)$. Intrusive thoughts were additionally reported from individuals with restrictive disorders $(\beta=0.12$, $\mathrm{p}=0.032$ ).

Overall, the models attained small effect sizes $\left(f^{2}=0.04\right.$ for depression, $\mathrm{f}^{2}=0.14$ for anxiety).$^{23}$ All models were significant.

\section{DISCUSSION}

The present study aimed at testing whether non-malignant ARDs and lung function impairment (ie, obstruction and restriction) have differential effects on mental health and psychological strain outcomes of formerly asbestos-exposed workers of the power industry in Germany. Considering the two potentially influencing factors simultaneously, results indicate that the mere knowledge of an existing non-malignant ARD is associated with psychological strain only but not with generalised psychological health disorders like anxiety or depression. These mental health outcomes were rather associated with an obstructive lung function impairment. Obstruction represents a potentially perceivable bodily health impairment, which can be assumed to be present throughout the day of the affected individual and not as easily neglected or denied as the knowledge of a past event.

Still, according to the depression screening scale, with $2.8 \%$ the value for the point prevalence of depression was lower in comparison to both the prevalence rate of the general male population in Germany $(4.2 \%)$ and prevalence reports of similar studies $(9.9 \%) .{ }^{19}$ In addition, participants reported less depressive symptoms than patients suffering from COPD or IPF. ${ }^{12} 13$ Similarly, the point prevalence of an anxiety disorder was lower $(10.6 \%)$ in comparison to prior studies on asbestos workers $(19.7 \%)$ but still twice as high as those of the general population. ${ }^{19}$

One reason for the rather good mental health findings of study participants might lie in the measure of symptom assessment in previous studies. A recent study has compared the validity of the DASS scales with the frequently used HADS scale and reported reduced sensitivity and specificity of the HADS scale in comparison to the DASS scale for depression. The DASS scale has been specifically designed for the assessment of depression and anxiety independent from overlapping symptoms of mental and somatic illnesses. Therefore, other scales might have overestimated potential mental health

Table 3 Point prevalence (\%) and OR of mental health in relation to the diagnosis of a non-malignant ARD

\section{No-diagnosis of non-}

malignant ARD cases (\% Diagnosis of non-malignant

out of total $N$ ) ARD cases (\% out of total $N)$

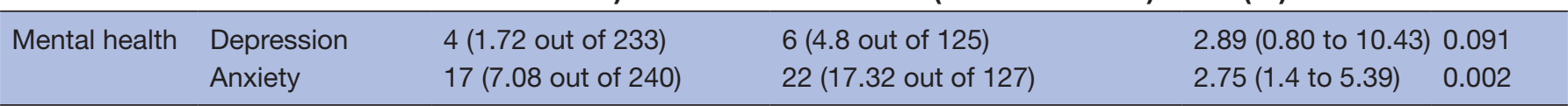

All scales are dichotomised according to scale cut-off values.

ARD, asbestos-related disease. 
Table 4 Hierarchical multiple regression analyses of lung function impairments and risk knowledge on mental health and psychological strain outcomes

\begin{tabular}{|c|c|c|c|c|c|c|c|c|c|c|c|c|}
\hline \multirow{3}{*}{$\begin{array}{l}\text { Outcome variables } \\
\text { Predictor variables }\end{array}$} & \multicolumn{6}{|c|}{ Mental health outcomes } & \multicolumn{6}{|c|}{ Psychological strain outcomes } \\
\hline & \multicolumn{3}{|c|}{ Depression } & \multicolumn{3}{|c|}{ Anxiety } & \multicolumn{3}{|c|}{$\begin{array}{l}\text { Specific fear of } \\
\text { cancer }\end{array}$} & \multicolumn{3}{|c|}{ Intrusion } \\
\hline & $\beta$ & $\mathbf{T}$ & $P$ value & $\beta$ & $\mathbf{T}$ & $P$ value & $\beta$ & $\mathbf{T}$ & $P$ value & $\beta$ & $\mathbf{T}$ & $P$ value \\
\hline $\begin{array}{l}\text { Diagnosis of non- } \\
\text { malignant ARDs }\end{array}$ & -0.05 & -0.78 & 0.435 & 0.07 & 1.10 & 0.272 & 0.13 & 2.01 & 0.046 & 0.17 & 2.99 & 0.003 \\
\hline Obstruction & 0.18 & 2.93 & 0.004 & 0.22 & 3.78 & $<0.001$ & 0.01 & 0.20 & 0.841 & 0.05 & 0.99 & 0.323 \\
\hline Restriction & 0.00 & -0.02 & 0.988 & 0.04 & 0.69 & 0.491 & 0.06 & 0.93 & 0.356 & 0.12 & 2.15 & 0.032 \\
\hline $\begin{array}{l}\text { Adjusted } R^{2} \text { of the } \\
\text { model }\end{array}$ & 0.04 & & & 0.12 & & & 0.06 & & & 0.10 & & \\
\hline$F$ value & 2.84 & & 0.011 & 7.68 & & $<0.001$ & 4.07 & & 0.001 & 6.78 & & $<0.001$ \\
\hline Total $\mathrm{N}$ in the model & 290 & & & 297 & & & 271 & & & 326 & & \\
\hline
\end{tabular}

All analyses are controlled by age, education and cumulative asbestos exposure.

ARDs, asbestos-related diseases.

symptoms in their sample, since somatic comorbidities in a rather elderly sample cannot be excluded. ${ }^{19}$

The occurrence of psychological symptoms may also have been determined by the knowledge of the planned preventive examination. Single studies have shown that the extensive preventive medical check-up can lead to psychological strain, which may be triggered by anticipation of the examination itself. ${ }^{34}$ Thus, when asking for the occurrence of psychological symptoms in the past 2 weeks, the symptoms could also be a cause of an acute adjustment disorder. An assessment of these symptoms independent from the medical examination would be necessary to exclude the acute effect.

The presence of psychological symptoms related to specific mental health disorders (ie, depression, anxiety) was solely related to ventilation disorders. Contrary, the presence of an acknowledged occupational disease, and thus the knowledge of an organic change that has already occurred due to past asbestos exposure particularly increased not the general but the specific fear of cancer due to the hazardous substance. This specific fear could probably trigger symptoms of general anxiety in individuals. Ultimately, the point prevalence for anxiety disorders was higher in the present sample than in the general male population. After all, these two variables are highly correlated (Spearman $r=0.33, \mathrm{p}<0.001$ ) with each other in the present sample, suggesting that specific fear of asbestos-related cancer might mediate the association between the diagnosis and anxiety symptoms.

In addition, the knowledge of a non-malignant ARD impairs the psychological state of mind to such an extent that it prevents those affected from switching off mentally by intrusive thoughts of the exposure. These intrusive thoughts might also be relevant for mental health disorders since intrusion has been linked to the development of depressive symptoms. ${ }^{24}$ Since we did not realise a longitudinal design in the present study, we could not test such mediation effects, which would need to infer that one mental health state (ie, specific fear or intrusive thoughts) would have preceded the other (ie, anxiety or depressive symptoms).

The strength of the present study is to combine measures from different sources (medical assessments, self-reported surveys) for predicting psychological wellbeing, reducing the potential for monomethod bias. However, one limitation was the low rate of participation in the psychological screening survey, possibly due to the length of the questionnaire, and the advanced age and physical well-being of the study participants, as our group comparison suggested. The older the participants were and the more they suffered from obstructive disorders, the less likely were they to fill out the additional psychological survey presented to them at their attendance of the health surveillance screening. In fact, previous studies have shown that advanced age but not health status per se is a main predictor for refusal of screening programme. ${ }^{25}$ Potential reasons for participants' refusal can be derived from those individuals who took the opportunity to fill out the open response possibilities of the survey. The majority of the individuals remarked that they either did not have an interest in the psychological research question but also that the survey required too much effort from them. This increased effort might have been one hindrance factor for participants with an obstructive health impairment. Since restriction was much less common and less severe compared with obstruction in the sample, the respective impairment might have not carried a significant weight. Still, when asked how satisfied study participants were with the health surveillance programme in general, overall the large majority reported to be satisfied $(57.9 \%)$ and very satisfied $(25 \%)$ with the health surveillance programme.

Another potential limitation related to the psychological health outcomes is that only male participants attended the health surveillance programme in the present study, a fact that is due to the specific study population of formerly asbestos-exposed workers. The majority 
of past studies on psychological distress and asbestos mainly included men. ${ }^{6}$ In general, female gender is associated with an increased risk of mental disorders ${ }^{8}$ and the majority of past studies on asbestos-exposed workers have also reported poorer psychological health of female participants than male participants. ${ }^{1}$ Thus, future studies on psychological effects following hazardous substance exposure (besides asbestos) would need to replicate the present findings of differential effects on knowledge versus functional impairment for generalisability reasons.

Finally, the effect size indices of the present study (ie, partial regression coefficients and explained variance) are small ${ }^{23}$ having made the findings detectable mainly by the relatively large sample size. However, it has to be considered that past studies who found stronger effects solely relied on self-report data on asbestos exposure and risk perception as well as on psychological health reports. ${ }^{1326}$ Other studies who were not able to detect any effects were also based on more objective criteria like radiological abnormalities. ${ }^{10}$ Therefore, the small but detectable impact of our findings using objective medical data as predictors can be considered of relevance. Of course, psychological disorders like depression are determined by multiple factors (eg, individual differences in genetics, neurobiology and social background) leaving enough room for third variables to explain more variance not considered in the present study. ${ }^{27}$

As a practical implication, it would be advisable for physicians and examiners to be aware of the influence of functional impairments on the psychological well-being of participants in the programme. Functional health impairments increase the risk for mental health problems ${ }^{13}$ which in turn have the potential to worsen the overall health status. Thus, screening for mental health symptoms could be included in routine medical screening examinations to detect a potential need of psychological support among programme participants. In fact, a recent review called for an interdisciplinary intervention for people with past asbestos exposure including a psychological assessment. ${ }^{2}$

As a theoretical implication, future research should longitudinally assess the cognitive processes that evolve after a diagnosis following hazardous substance exposure, which at the same time carries the message of an increased future health risk. Past research on risk communication has shown that psychological symptoms only appear immediately after providing the health threatening information but fade away even within a short period of time. ${ }^{7}$ Thus, there must be some coping mechanism behind the information processing of an increased health risk, which support individuals to maintain their psychological well-being. From the present study, it seems as if affected individuals mentally and emotionally continue to deal with their specific health risk even years after exposure cessation but that the individual strain does not spread out to a generalised mental health disorder unless there is a bodily perceivable change on the individuals' health status potentially related to the past asbestos exposure.
To better understand the underlying mechanisms and set this research on sound theoretical grounds, future research could rely on past models from fear appeal research (see ${ }^{28}$ for an overview).

Acknowledgements The authors thank the whole team of the outpatient clinic of the study centre for their effort in data collection. We also thank Christian Schikowsky for database management, Dr André Esser for providing the lung function variables according to the reference values and Kathy Bischof for proofreading the manuscript for language and grammar.

Contributors All authors declare that they provided substantial contributions to the conception or design of the work, and the acquisition, analysis and interpretation of data for the work. JL drafted the work and MKF and TK revised it critically for important intellectual content.

Funding This project was funded by the employer's liability insurance association in the Energy, Textile, Electrical, and Media Industry (BGETEM), Cologne, Germany, with an unrestricted grant to the Uniklinik RWTH Aachen University.

Competing interests None declared.

Patient consent for publication Not required.

Ethics approval The present study was approved by the independent ethics committee from the medical faculty of the university's study centre under the number EK 043/09.

Provenance and peer review Not commissioned; externally peer reviewed. Data availability statement Data are available on reasonable request.

Open access This is an open access article distributed in accordance with the Creative Commons Attribution Non Commercial (CC BY-NC 4.0) license, which permits others to distribute, remix, adapt, build upon this work non-commercially, and license their derivative works on different terms, provided the original work is properly cited, appropriate credit is given, any changes made indicated, and the use is non-commercial. See: http://creativecommons.org/licenses/by-nc/4.0/.

ORCID iD

Jessica Lang http://orcid.org/0000-0001-7802-8546

\section{REFERENCES}

1 Mounchetrou Njoya I, Paris C, Dinet J, et al. Anxious and depressive symptoms in the French asbestos-related diseases cohort: risk factors and self-perception of risk. Eur J Public Health 2017;27:359-66.

2 Bonafede M, Ghelli M, Corfiati M, et al. The psychological distress and care needs of mesothelioma patients and asbestos-exposed subjects: a systematic review of published studies. Am J Ind Med 2018;61:400-12.

3 Maurel M, Stoufflet A, Thorel L, et al. Factors associated with cancer distress in the asbestos post-exposure survey (APEXS). Am J Ind Med 2009;52:288-96.

4 Paris C, Maurel M, Luc A, et al. CT scan screening is associated with increased distress among subjects of the APExS. BMC Public Health 2010;10:647.

5 Lebovits AH, Byrne M, Strain JJ. The case of asbestos workers: A psychological evaluation. In: Lebovits AH, Baum A, Singer JE, eds. Advances in environmental psychology. Hillsdale, NJ: Lawrence Erlbaum Associates, Inc, 1986: Vol 6. 3-17.

6 Barak Y, Achiron A, Rotstein Z, et al. Stress associated with asbestosis: the trauma of waiting for death. Psychooncology 1998;7:126-8.

7 Shaw C, Abrams K, Marteau TM. Psychological impact of predicting individuals' risks of illness: a systematic review. Soc Sci Med 1999;49:1571-98.

8 World Health Organisation. The ICD-10 classification of mental and behavioural disorders. Clinical descriptions and diagnostic guidelines. Geneva: World Health organization, 1993. Available: http://www.who.int/classifications/icd/en/bluebook.pdf [Accessed 26 Feb 2018].

9 Jacobi F, Wittchen H-U, HÖLTING C, et al. Prevalence, co-morbidity and correlates of mental disorders in the general population: results from the German health interview and examination survey (GHS). Psychol Med 2004;34:597-611.

10 Franklin P, Robinson M, Abaogye-Sarfo P, et al. The mental health of asbestos-exposed subjects with pleural abnormalities. Int Arch Occup Environ Health 2015;88:343-50. 
11 Wolff $\mathrm{H}$, Vehmas T, Oksa P, et al. Asbestos, asbestosis, and cancer, the Helsinki criteria for diagnosis and Attribution 2014: recommendations. Scand J Work Environ Health 2015;41:5-15.

12 Maurer J, Rebbapragada V, Borson S, et al. Anxiety and depression in COPD: current understanding, unanswered questions, and research needs. Chest 2008;134:43S-56.

13 Matsuda T, Taniguchi H, Ando M, et al. Depression is significantly associated with the health status in patients with idiopathic pulmonary fibrosis. Intern Med 2017;56:1637-44.

14 Felten MK, Knoll L, Eisenhawer C, et al. Retrospective exposure assessment to airborne asbestos among power industry workers. $J$ Occup Med Toxicol 2010;5.

15. . BK-Report 1/2013 Faserjahre: Berufsgenossenschaftliche Hinweise zur Ermittlung der kumulativen AsbestfaserstaubDosis am Arbeitsplatz (Faserjahre) und Bearbeitungshinweise zur Berufskrankheit Nr. 4104 "Lungenkrebs oder Kehlkopfkrebs". [Employers' liability insurance association notes on the determination of the cumulative dose of asbestos fibre dust at the workplace (fibre years) and processing notes on occupational disease No. 4104 "Lung cancer or throat cancer"]; 2013. https://publikationen.dguv.de/ forschung/ifa/ifa-report/2757/faserjahre-bk-report-1/2013

16 Quanjer PH, Stanojevic S, Cole TJ, et al. Multi-Ethnic reference values for spirometry for the 3-95-yr age range: the global lung function 2012 equations. Eur Respir J 2012;40:1324-43.

17 Lovibond SH, Lovibond PF. Manual for the depression anxiety stress scales. 2nd ed. Sydney, Australia: Psychological Foundation of Australia, 1995

18 Antony MM, Bieling PJ, Cox BJ, et al. Psychometric properties of the 42 -item and 21 -item versions of the Depression Anxiety Stress
Scales in clinical groups and a community sample. Psychol Assess 1998;10:176-81.

19 Nilges P, Essau C. Die Depressions-Angst-Stress-Skalen [The Depression Anxiety Stress Scales]. Schmerz [Pain] 2015;29:649-57.

20 Berman SH, Wandersman A. Measuring knowledge of cancer. Soc Sci Med 1991;32:1245-55.

21 Horowitz M, Hulley S, Alvarez W, et al. News of risk for early heart disease as a stressful event. Psychosom Med 1980;42:37-46.

22 Corp IBM. IBM SPSS statistics for windows, version 25.0. 2017. Armonk (NY), USA: IBM Corp, 2017.

23 Cohen J. A power primer. Psychol Bull 1992;112:155-9.

24 Smets J, Wessel I, Schreurs E, et al. The interplay between rumination and intrusions in the prediction of concurrent and prospective depressive symptoms in two nonclinical samples. Psychol Rec 2012;62:777-88.

25 Knoll L, Felten MK, Ackermann D, et al. Non-Response bias in a surveillance program for asbestos-related lung cancer. J Occup Health 2011;53:16-22.

26 Lin Q-H, Jiang C-Q, Lam T-H, et al. Past occupational dust exposure, depressive symptoms and anxiety in retired Chinese factory workers: the Guangzhou Biobank cohort study. J Occup Health 2014;56:444-52.

27 Cattapan-Ludewig K, Seifritz E. [Aetiology of depressive disorders-the biopsychosocial model]. Ther Umsch 2010;67:566-70.

28 Kok G, Peters G-JY, Kessels LTE, et al. Ignoring theory and misinterpreting evidence: the false belief in fear appeals. Health Psychol Rev 2018;12:111-25. 